\title{
Benign versus malignant parakeratosis: a nuclear morphometry study
}

\author{
Jie Song ${ }^{1}$ and Christopher R Shea ${ }^{2}$ \\ ${ }^{1}$ Department of Pathology, University of Chicago Medical Center, Chicago, IL, USA and ${ }^{2}$ Section of \\ Dermatology, Department of Medicine, University of Chicago Medical Center, Chicago, IL, USA
}

\begin{abstract}
Parakeratosis is relatively common in both benign and malignant skin diseases. It is a useful feature for classifying certain types of dermatitis; however, its value in distinguishing benign from malignant neoplastic squamous lesions has not been investigated. Parakeratotic nuclei are pyknotic and often elongated, regardless of the underlying disease. However, we have noticed a quite consistent difference in nuclear morphology between benign and malignant parakeratosis, which has not been formally described in the literature. To test the hypothesis that morphological differences may aid in the discrimination between certain benign and malignant dermatopathological lesions, we used computer-aided nuclear morphometry to analyze parakeratosis from 28 cases of squamous cell carcinoma, 19 cases of verruca vulgaris, and 23 cases of psoriasiform dermatitis. Significant differences in nuclear area and area variability were observed between benign and malignant cases. These two parameters together could separate most squamous cell carcinomas from benign cases. Through this study, we hope to raise the awareness that even parakeratosis, the most superficial morphological datum available for histopathological evaluation, may harbor distinct changes of nuclear atypia in squamous cell carcinoma. In some overly superficial shave biopsies, parakeratosis may be the only material available for evaluation. For such cases, if squamous cell carcinoma is in the differential diagnosis, then the parakeratotic nuclei should be examined carefully to determine the likelihood of malignancy. Computer-aided nuclear morphometry may have potential diagnostic value for such cases.

Modern Pathology (2010) 23, 799-803; doi:10.1038/modpathol.2010.52; published online 26 February 2010
\end{abstract}

Keywords: parakeratosis; nuclear morphometry; squamous cell carcinoma

Parakeratosis is defined as the presence of nucleated keratinocytes in the stratum corneum, and is thought to be due to accelerated keratinocytic turnover. ${ }^{1}$ Parakeratosis may occur in both benign skin diseases (such as various dermatitides) and malignant neoplasms (such as squamous cell carcinoma). It serves as a useful morphological feature in differentiating and classifying certain inflammatory skin diseases. In contrast, whether parakeratosis has any value in the differential diagnosis between benign and malignant lesions is not clear. In our practice, we have noticed a quite consistent morphological difference between benign and malignant parakeratosis. Even though parakeratosis always has

Correspondence: Dr CR Shea, MD, Section of Dermatology, Department of Medicine, University of Chicago Medical Center, 5841 S. Maryland Avenue, MC 5067, Chicago, Illinois 60637, USA.

E-mail: cshea@medicine.bsd.uchicago.edu

Received 13 November 2009; revised 1 February 2010; accepted

3 February 2010; published online 26 February 2010 pyknotic and often elongated nuclei, regardless of the underlying disease, the parakeratotic nuclei of many malignant cases often appear more atypical, with larger and more irregular nuclei. This difference has not been formally described in the literature to our knowledge.

Nuclear morphometry provides objective measurements of nuclei, often assisted by digital imaging and specialized computer software. Nuclear morphometry has been shown to be accurate and potentially useful in aiding diagnosis and predicting prognosis in a variety of cancers, ${ }^{2-4}$ including cutaneous squamous cell carcinoma and its precursors. $^{5-7}$ This technique has also been used to analyze parakeratosis in psoriasis. ${ }^{8}$

The goal of this study is to test the hypothesis that morphological differences in parakeratosis may aid in the discrimination between certain benign and malignant dermatopathological lesions, as assessed through computer-aided nuclear morphometry. There is potential clinical significance in this study. For example, shave biopsy is one of the most 
popular techniques in dermatology because of its convenience and minimal injury. However, shave biopsies sometimes have inadequate depth, causing diagnostic challenges., ${ }^{9,10}$ Overly superficial shave biopsies composed mainly of parakeratosis would probably be non-diagnostic, but they may still provide valuable information if a consistent morphological difference between benign and malignant parakeratosis can be proven. This information may further guide clinical decisions such as how decisively a re-biopsy is needed.

\section{Materials and methods}

\section{Case Selection}

All skin biopsy specimens accessioned from January to June 2008 at the dermatopathology service of the University of Chicago Medical Center were searched for the following terms in the final diagnosis: squamous cell carcinoma, verruca vulgaris, and psoriasis/psoriasiform dermatitis. These cases were retrieved and reviewed, and only the ones that contained a significant amount of parakeratosis $(\geq 3$ high-power fields, with $\geq 30$ parakeratotic nuclei in each field by visual estimation) were selected. A total of 28 cases of squamous cell carcinoma, 19 cases of verruca vulgaris, and 23 cases of psoriasiform dermatitis were included in this study. Institutional review board approval for this study was granted.

\section{Digital Imaging}

Digital images were taken at $\times 400$ magnification, $1300 \times 1030$ pixels ( 1 pixel $=0.22 \mu \mathrm{m}$ ), using an Olympus BX41 microscope, an AxioCam (Carl Zeiss) digital camera, and an Intel computer (2.4 GHz, 2 Gb RAM) running AxioVision LE image acquisition software (Carl Zeiss). The same settings for camera, microscope, and software were used for all cases. For cases with relatively small areas of parakeratosis, three to five images were taken to encompass the entire parakeratosis. For cases with larger areas of parakeratosis, we took five images evenly distributed along the epidermis in order to minimize the bias. Foci with abundant inflammatory cells, yeasts, or other artifacts were skipped, as they may be mistaken as nuclei by image analysis software. The raw images were then manually edited using Photoshop 6.0 (Adobe Systems) to remove non-parakeratotic areas.

\section{Nuclear Morphometry}

Image segmentation, nuclear identification, and nuclear morphometry were performed using the automated open-source program CellProfiler (http:// www.cellprofiler.org). ${ }^{11}$ The original color images were converted into grayscale images. Segmentation was performed by the program's automatic thresh- olding algorithm, to identify objects that were darker than the surrounding environment; these objects were potential nuclei. Filters were used to exclude objects that were either too big or too small, which usually represented various staining artifacts, overlapping nuclei or nuclear debris. Accepted nuclei were highlighted by white borders and printed in output JPEG files (Figure 1). These output images were manually inspected as a measure of quality assurance, and they also provided guidance for optimizing the program settings. For each nucleus, five basic parameters were measured: nuclear area, perimeter, major axis, minor axis, and form factor. Form factor is calculated by the formula $\left(4 \times \mathrm{pi} \times\right.$ area/perimeter $\left.{ }^{2}\right)$ and is a measurement of 'roundness.' It equals unity (1) for perfectly round objects, and decreases to 0 for more irregular or elongated objects.

Selected cases were also analyzed by ImagePro 6.0 (Media Cybernetics). Image segmentation was done by manually picking a few representative nuclei, which served as color references and were processed by the program's proprietary algorithm (Figure 2). The same nuclear parameters as described above were measured with ImagePro.

\section{Statistical Analysis}

Each case had multiple nuclei identified and measured by computer programs; therefore, for each case, 10 parameters were calculated, which included the mean and standard deviation of each basic nuclear parameter. The standard deviation was defined as 'variability' in this study, because it basically reflects the degree of variation among the nuclei of a single case. Two-tailed Student's $t$ test was used to compare the statistical significance of differences among different diagnostic groups.

\section{Results}

Each case had at least 100 parakeratotic nuclei identified by CellProfiler (average 337, range 126768). When comparing the three diagnostic groups, we identified four parameters that showed at least 1.5-fold difference in their means between squamous cell carcinoma and verruca vulgaris/ psoriasiform dermatitis: nuclear area, area variability, perimeter variability, and minor axis variability (Table 1). All these parameters yielded $P$ values $<0.001$, on comparing squamous cell carcinoma with verruca vulgaris, or squamous cell carcinoma with psoriasiform dermatitis. There was no significant difference between verruca vulgaris and psoriasiform dermatitis for any parameter.

Nuclear area was particularly interesting because it showed significant difference in both mean (average nuclear size of a case) and variability (nuclear size variation of a case). This indicates that cases of malignant parakeratosis had not only larger 


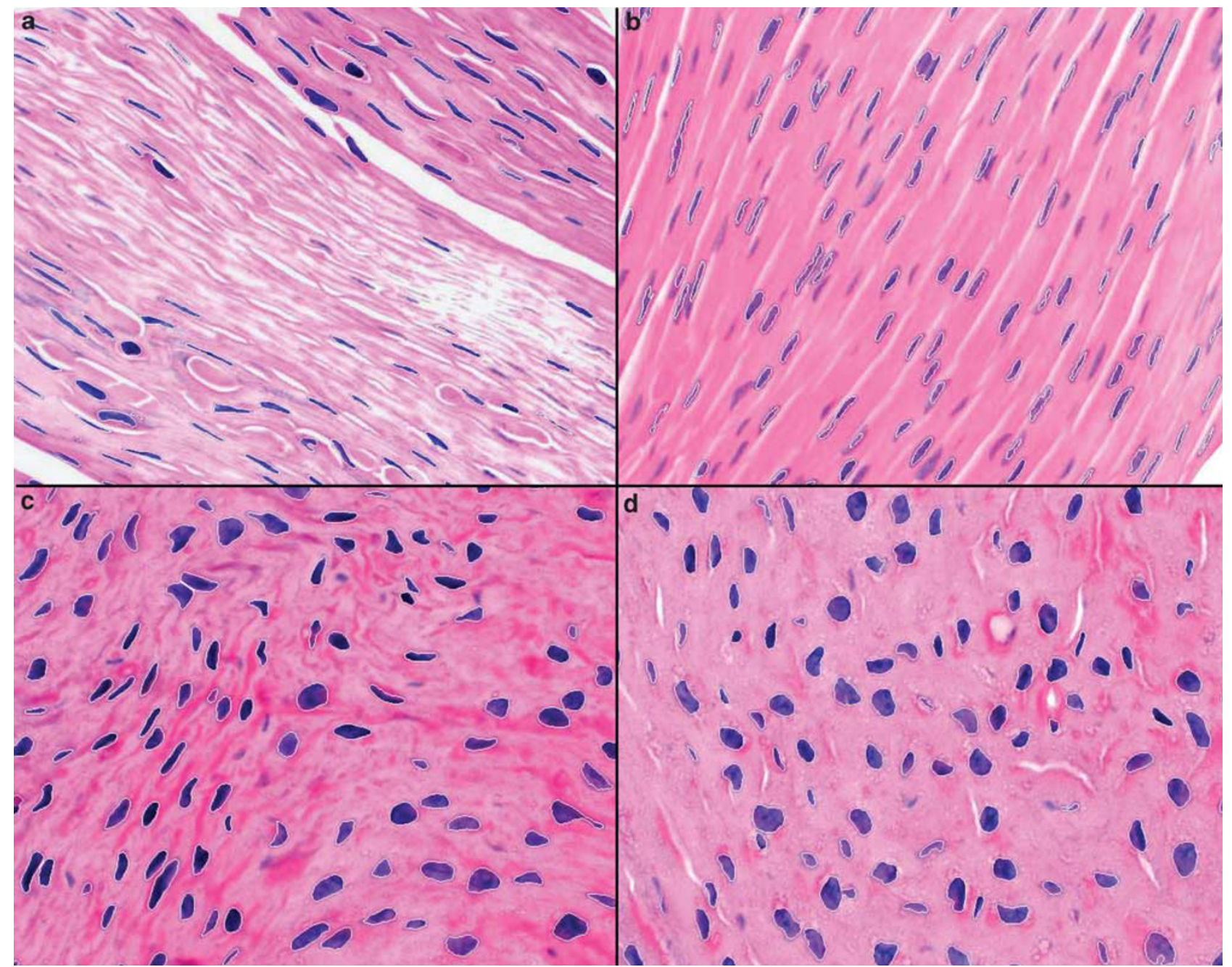

Figure 1 CellProfiler snapshots. Examples of psoriasiform dermatitis (a and b) and squamous cell carcinoma (c and d) processed by CellProfiler, with nuclei highlighted with white borders.

nuclei, but also a higher degree of variation in nuclear size. This is consistent with what we have observed by inspection in practice. In fact, nuclear area and area variability together were able to distinguish most cases of benign from malignant parakeratosis (Figure 3). However, some overlap does exist, and some squamous cell carcinoma cases could not be distinguished from benign cases by these two parameters alone.

There exists no 'gold standard' for nuclear morphometry. In order to validate the results of CellProfiler, 10 cases from each diagnostic group were randomly selected and analyzed by ImagePro, as described above. The parameters were then compared case-by-case. Correlation coefficients between these two programs were very strong for all parameters $(r>0.9, P<0.001)$, suggesting that both programs provided reasonably accurate results.

\section{Discussion}

Nuclear morphometry has been proven to be a useful tool for the diagnosis and prediction of prognosis in squamous cell carcinoma and precursor lesions of skin and mucosa. ${ }^{4,6,12,13}$ Previous studies analyzed only viable, non-parakeratotic cells. Parakeratosis is not uncommon in squamous cell carcinoma; ${ }^{14}$ however, its value in distinguishing squamous cell carcinoma from benign lesions has apparently not been investigated previously.

In this study, we used computer-aided nuclear morphometry to prove that there is a significant morphological difference between benign and malignant parakeratosis, confirming what we have observed in practice. This distinction, which has not been previously described in the literature, may have been overlooked because pyknotic, parakeratotic nuclei are usually not optimal objects for investigation by light microscopy. However, we consider it worthwhile to raise awareness of this morphological distinction, which may be particularly useful for overly superficial shave biopsies composed entirely or mostly of parakeratosis. For such specimens, if squamous cell carcinoma is a consideration, careful examination of the 


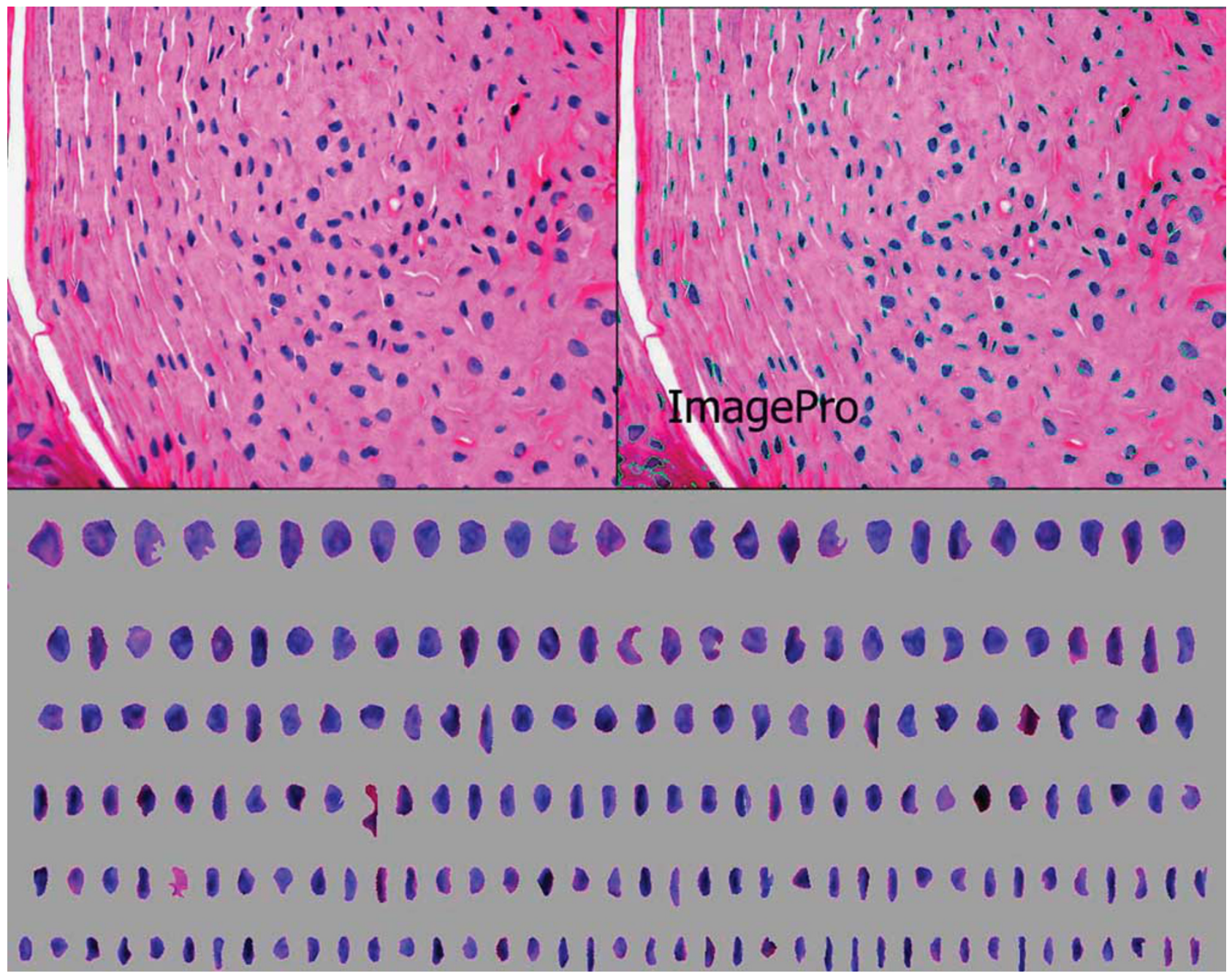

Figure 2 ImagePro snapshots. A typical squamous cell carcinoma case is shown with the original image (upper left), and ImagePro processed image with nuclei highlighted with green borders (upper right). The nuclei are also shown individually (lower).

Table 1 Average parameters of different diagnostic groups

\begin{tabular}{|c|c|c|c|c|c|c|c|c|c|c|}
\hline \multirow[t]{2}{*}{ Parameters } & \multicolumn{2}{|c|}{ Area } & \multicolumn{2}{|c|}{ Perimeter } & \multicolumn{2}{|c|}{ Major axis } & \multicolumn{2}{|c|}{ Minor axis } & \multicolumn{2}{|c|}{ Form factor } \\
\hline & $M$ & $V$ & $M$ & $V$ & $M$ & $V$ & $M$ & $V$ & $M$ & $V$ \\
\hline Psoriasiform dermatitis $(n=23)$ & 238 & 96 & 79 & 19 & 35 & 8 & 8 & 1 & 0.46 & 0.11 \\
\hline Verruca vulgaris $(n=19)$ & 211 & 91 & 72 & 17 & 31 & 8 & 8 & 2 & 0.5 & 0.14 \\
\hline Squamous cell carcinoma $(n=28)$ & 341 & 183 & 89 & 27 & 37 & 11 & 11 & 3 & 0.53 & 0.14 \\
\hline
\end{tabular}

Abbreviations: M, mean; V, variability.

Columns highlighted in bold represent parameters with more than 1.5 -fold difference between squamous cell carcinomas and benign diseases. Unit $=$ pixel.

parakeratotic nuclei could help to determine the likelihood of malignancy. Even though a definite diagnosis cannot and must not be rendered on an inadequate specimen, this evaluation could nonetheless provide useful information about the nature of the lesion. For example, if the nuclei are overtly atypical, the likelihood of malignancy would be high and a re-biopsy may be indicated.
This study also suggests the potential value of computer-aided nuclear morphometry in the histopathologic diagnosis of squamous cell carcinoma. Two simple nuclear parameters, area and area variability, were able to distinguish most squamous cell carcinomas from benign cases in our study. Some overlap between these two groups did exist; however, more morphometric parameters could be 


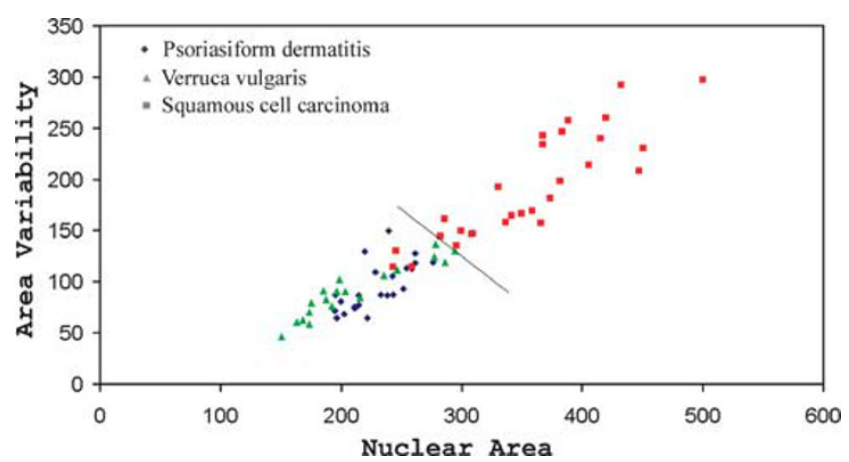

Figure 3 Nuclear area and area variability together were able to distinguish most squamous cell carcinomas from benign cases. Squamous cell carcinomas (red squares) are clustered in the upper right corner, whereas psoriasiform dermatitis (blue diamonds) and verruca vulgaris (green triangles) cases are in the lower left corner, except for rare exceptions. Unit = pixel

measured and might provide additional criteria for this differential diagnosis. Parameters that quantify the relationship between neighboring nuclei, such as their relative orientation and organization, might prove particularly useful. Prospective studies on unknown cases may be interesting to further investigate the diagnostic value of morphometry.

\section{Disclosure/conflict of interest}

The authors declare no conflict of interest.

\section{References}

1 Lever WF, Elder DE. Lever's Histopathology of the Skin, 9th edn. Lippincott Williams \& Wilkins: Philadelphia, 2005.

2 Fleming MG, Rauber TW. Multiparametric image cytometry in mycosis fungoides. J Invest Dermatol 1996;106:129-134.
3 Gil J, Wu H, Wang BY. Image analysis and morphometry in the diagnosis of breast cancer. Microsc Res Tech 2002;59:109-118.

4 Pektas ZO, Keskin A, Gunhan O, et al. Evaluation of nuclear morphometry and DNA ploidy status for detection of malignant and premalignant oral lesions: quantitative cytologic assessment and review of methods for cytomorphometric measurements. J Oral Maxillofac Surg 2006;64:628-635.

5 Narvaez D, Kanitakis J, Euvrard S, et al. Comparative nuclear morphometric analysis of aggressive and nonaggressive squamous cell carcinomas of the skin. Acta Dermatol Venereol 1997;77:115-117.

6 Carpenter PM, Linden KG, McLaren CE, et al. Nuclear morphometry and molecular biomarkers of actinic keratosis, sun-damaged, and nonexposed skin. Cancer Epidemiol Biomarkers Prev 2004;13:1996-2002.

7 Lavie O, Maini I, Pilip A, et al. Computerized nuclear morphometry for the prediction of inguinal lymph nodes metastases in squamous cell carcinoma of the vulva. Int J Gynecol Cancer 2006;16:556-561.

8 Johannesson A, Hammar H. Corneocyte morphology and formation rate in lichen planus and experimental parakeratosis in subjects with and without psoriasis. Acta Dermatol Venereol 1982;62:463-470.

9 Kopf AW, Popkin GL. Letter: shave biopsies for cutaneous lesions. Arch Dermatol 1974;110:637.

10 Stell VH, Norton HJ, Smith KS, et al. Method of biopsy and incidence of positive margins in primary melanoma. Ann Surg Oncol 2007;14:893-898.

11 Carpenter AE, Jones TR, Lamprecht MR, et al. CellProfiler: image analysis software for identifying and quantifying cell phenotypes. Genome Biol 2006;7:R100.

12 Sekine J, Uehara M, Hideshima K, et al. Predictability of lymph node metastases by preoperative nuclear morphometry in squamous cell carcinoma of the tongue. Cancer Detect Prev 2003;27:427-433.

13 Giardina C, Caniglia DM, D'Aprile M, et al. Nuclear morphometry in squamous cell carcinoma (SCC) of the tongue. Eur J Cancer B Oral Oncol 1996;32B:91-96.

14 Resnik KS, DiLeonardo M. Incidental granular parakeratotic cornification in carcinomas. Am J Dermatopathol 2007;29:264-269. 